\title{
Hoch dosierte Therapie für höchste Ansprüche
}

$\mathrm{H}$ och dosierte Sublingualpräparate der neuesten Generation werden bereits mit guten Ergebnissen in der Praxis eingesetzt. Entsprechend positiv bewertet auch die von den deutschen allergologischen Fachgesellschaften gerade aktualisierte Leitlinie zur Hyposensibilisierung (Allergo J 2009; 18 : 508-37) die Datenlage zur Wirksamkeit der sublingualen Immuntherapie (SLIT). So bewirken hoch dosierte Präparate ähnliche Immuneffekte wie die klassische subkutane Immuntherapie. Die Leitlinie befürwortet daher den Einsatz der SLIT bei der allergischen Rhinokonjunktivitis unter der Voraussetzung, dass die klinische Wirksamkeit des eingesetzten Präparates durch doppelblinde und plazebokontrollierte Studien belegt ist.

$\mathrm{Zu}$ den hoch dosierten Präparaten zählt auch AllerSlit ${ }^{\circledR}$ forte Gräser, das laut CREATE-Assay einen Allergengehalt von 21,6 $\mu \mathrm{g}$ Gräser-Gruppe-5-Allergen $(40 \mu \mathrm{g}$ im Inhouse-Assay) in der Erhaltungsdosis aufweist. Das flüssige Einzeldosispräparat zeigte in einer multizentrischen, randomisierten und plazebokontrollierten Doppelblindstudie gute Ergebnisse bei Wirksamkeit und Verträglichkeit (Eur Ann Allergy Clin Immunol 2006; 38: 355-60). In der Untersuchung hatten 188 Teilnehmer die durch die Allergie verursachten Symptome und die entsprechende Medikation in Patiententagebüchern dokumentiert. Insgesamt umfasste der Zeitraum der Studie drei Pollenflugphasen. Bereits nach 3-4 Monaten Therapie war es zu einer deutlichen Reduktion der Symptome und der benötigten Medikation gekommen. Der Vergleich der Ergebnisse im kombinierten Symptom-Medikations-Score ergab einen Unterschied von 46\% Prozent zwischen Verum und Plazebo.

Über die Wirksamkeit und Verträglichkeit hinaus sollte sich ein Sublingualpräparat durch praktische Handhabung und schnelle Aufdosierung auszeichnen. Auch diesen Anforderungen entspricht AllerSlit ${ }^{\circledR}$ forte Gräser. Die Hochdosis-SLIT ist innerhalb einer halben Stunde durch ein Verdoppeln der Dosen mit Wartezeiten von jeweils 15 Minuten auftitrierbar. Die Dosissteigerung kann somit problemlos in den Praxisablauf integriert werden, wie eine aktuelle Anwendungsstudie mit Allergologen gezeigt hat: 95\% der Teilnehmer bestätigten die Praktikabilität des Verfahrens.

Die Praktikabilität einer SLIT erleichtert nicht nur ihre Anwendung, sie unterstützt auch die Compliance, die für den Erfolg der Therapie Grundvoraussetzung ist. „Es ist wichtig, die Patienten vor Therapiebeginn ausführlich über die SLIT und ihre spätere selbstständige Anwendung zuhause zu informieren“, erklärt dazu Prof. Dr. Bertrand Gloddek, Meppen. „Neben dem intensiven Beratungsgespräch kann es hilfreich sein, die Patienten aufzufordern, sich bei Fragen auch kurzfristig in der Praxis zu melden. “ Um die Compliance zu verbessern, kann auch eine „vertragsähnliche" Vereinbarung über die Therapie abgeschlossen werden. Damit wird für den Patienten eine größere Verbindlichkeit geschaffen. Als weitere Maßnahmen sind ein erneutes Einbestellen in die Praxis vier bis sechs Wochen nach der Aufdosierung oder eine spätere Kontaktaufnahme per Telefon sinnvoll.

Bei längerer Einnahme der Hochleistungs-SLIT von Allergopharma profitieren die Patienten im besonderen $\mathrm{Maße}$, wie eine Studie gezeigt hat (Allergo J 2008; 17 [S2]: 88-9): Die Teilnehmer litten während der laufenden Pollensaison deutlich weniger unter Allergiesymptomen und benötigten keine Medikamente mehr. Die Anzahl der symptomarmen und medikationsfreien „Well Days“ während der Saison von 42 Tagen betrug im Median 49\% in der zweiten und $88 \%$ in der dritten Saison. Diese Ergebnisse zeigen, dass AllerSlit ${ }^{\circledR}$ forte Allergiekranken dabei hilft, ihre Lebensqualität kontinuierlich $\mathrm{zu}$ verbessern.

Nach Informationen von Allergopharma, Reinbek

\section{Starter-Set wieder da}

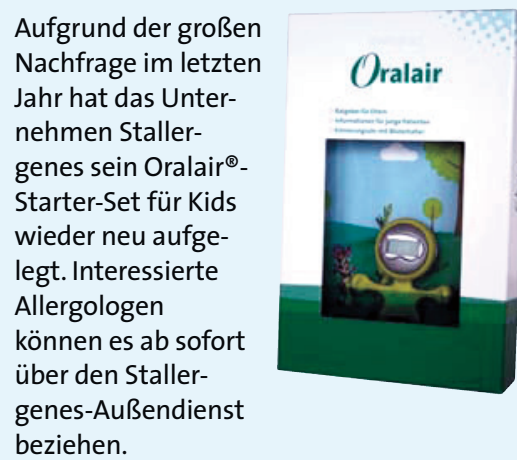

Bewährte Inhalte unterstützen die tägliche Praxis: Die Patientenbroschüre „Alles was du über deine Allergie und deine Behandlung mit Oralair ${ }^{\circledR}$ wissen solltest" erklärt kindgerecht die Hyposensibilisierungstherapie. Dazu gibt es eine Elternbroschüre mit Motivationstipps für das Kind, denn eine konsequente Einnahme ist entscheidend für den Therapieerfolg. So darf natürlich auch das grüne Uhrenmännchen „Flexi“ als symbolischer Unterstützer nicht fehlen. Flexi erinnert mittels einer Alarmfunktion an die tägliche Tabletteneinnahme. Und damit die Tabletten dann direkt griffbereit sind, kann der Blister mit einem Clip am Kopf des Uhrenmännchens befestigt werden.

Nach Informationen von Stallergenes, Kamp-Lintfort

\section{Nasenspray mal drei}

Der Generikahersteller Hexal bietet sein Budesonid-Nasenspray seit März neu in einer besonders niedrigen Dosierung an. Budes ${ }^{\circledR}$ Nasenspray $32 \mu \mathrm{g}$ ergänzt die bisher schon verfügbaren Wirkstärken $50 \mu \mathrm{g}$ und $64 \mu \mathrm{g}$ Budesonid pro Sprühstoß. Die große Dosierungspalette ermöglicht eine flexible und individuelle Therapie der saisonalen und perennialen allergischen Rhinitis. Die 32- $\mu$ g-Dosierung eignet sich besonders für Kinder ab sechs Jahren, aber auch für Erwachsene, bei denen eine geringere Dosis zur Symptomkontrolle ausreicht. Da das Spray nur einmal täglich angewendet werden muss, ist die Compliance bei den Patienten hoch.

Alle Budes ${ }^{\circledR}$ Nasensprays erlauben eine wirtschaftliche Therapie der allergischen Rhinitis. Die kostengünstigen Doppelpackungen bieten zusätzliches Einsparpotenzial in der Langzeitanwendung.

Nach Informationen von Hexal, Holzkirchen 